\title{
EL ENFOQUE COGNITIVO-COMPORTAMENTAL PARA LA ANSIEDAD POR LA SALUD ("HIPOCONDRÍA")
}

\author{
Paul M. Salkovskls y Katharine A. Rimes \\ University of Oxford Department of Psychiatry
}

\begin{abstract}
RESUMEN
El presente articulo describe las líneas generales del enfoque cognitivo aplicado a la ansiedad por la salud (hipocondría). En este trastomo, la ansiedad se operativiza mediante 4 factores cognitivos clave: la probabilidad percibida de tener una enfermedad; su "coste"; la percepción de la propia capacidad para afrontarla; y la percepción de cuánto ayudarán otros factores externos. Se plantea un modelo de mantenimiento de la ansiedad por la salud en el que varios factores como el arousal fisiológico, la malinterpretación de los sintomas, la atención selectiva a y el sesgo confirmatorio de la información relacionada con la enfermedad, y la conducta de comprobación, estarían manteniendo la ansiedad mediante el aumento de los sintomas y de la preocupación. A partir del modelo cognitivo-comportamental se exponen las líneas principales del tratamiento, cuya meta es que el paciente alcance la comprensión de cómo funciona su problema. Para ello se plantean explicaciones altemativas, se discute la evidencia a favor y en contra, y se llevan a cabo experimentos conductuales. Un aspecto que se destaca es el papel que juega, en el mantenimiento e incremento de la ansiedad, el hecho de tranquilizar a los pacientes. Por último se revisan los datos disponibles hasta la fecha acerca de la eficacia de este enfoque de tratamiento, y se sugieren líneas futuras de investigación.
\end{abstract}

Palabras clave: Ansiedad por la salud; hipocondria; enfoque cognitivo; tratamiento cognitivo-comportamental; tranquilización.

\section{ABSTRACT}

This paper describes the general features of the cognitive approach to health anxiety (hypochondriasis). The anxiety results from the interaction of four cognitive key factors: the perceived likelihood of iliness; its perceived cost; one's percelved ability to cope with the illness; and the perception of the degree in which external factors would help. A maintenance model of health anxiety is presented. In such a model several factors would be increasing both, physical symptoms and preoccupation about the problem. These hypothesised factors are: physiological arousal; misinterpretation of autonomic symptoms; selective attention to, and confirmatory bias of illness-related information; and checking behaviour. The basis of treatment is set from the general cognitivebehavioural approach. The aim of treatment is the patients' understanding of how their problem works. To reach such a goal, alternative explanations are provided, evidence for and against is discussed, and behavioural experiments are carried out. The role of reassurance is also highlighted as a feature that could be maintaining, and even increasing, health anxiety. Finally, the last data on the outcome of treatment research are reviewed, and future directions for research are suggested.

Key words: Health anxiety; hypochondriasis; cognitive approach; cognitivebehavioural treatment; reassurance.

Correspondencia: University of Oxford Department of Psychiatry. Wamerford Hospital, Oxford OX3 7JX. Reconocimiento: Paul Salkovskis es Wellcome Trust Senior Research Fellow.

Traducido por Myriam Gallardo Pérez. Licenciada en Psicologia. 
El rasgo esencial del diagnóstico de hipocondría es la preocupación por la creencia o el miedo de padecer una enfermedad grave. Ésto sucede sin que exista una patología orgánica adecuada que dé cuenta del grado de respuesta psicológica y "a pesar de la tranquilización médica”. Estos temores están asociados a la percepción de señales y sensaciones corporales que se malinterpretan como prueba de tener una enfermedad grave. Se ha propuesto que la hipocondría representa el extremo final de un continuo de ansiedad centrada en la salud (Salkovskis, 1989; Warwick y Salkovskis, 1989) presente en un amplio abanico de personas sin diagnóstico psiquiátrico, que incluye también a aquellos con enfermedad física.

Los pacientes que sufren de ansiedad por la salud consumen recursos de cada una de las áreas de la práctica médica (Katon et al., 1984; Kellner, 1985; Mayou, 1976; Warwick y Salkovskis, 1989). Durante muchos años se pensó que la hipocondría era siempre secundaria a otros trastornos, especialmente depresión (Kenyon, 1965). Estudios más recientes indican que la forma primaria del trastorno es común. Esta distinción es importante porque tiene implicaciones de primer orden para la evaluación y el manejo de los pacientes que presentan sintomas hipocondríacos.

La duración y el curso de la hipocondría son inciertos. La definición del DSM-IV de hipocondrla incluye un criterio de duración minima de 6 meses, pero sabemos poco acerca de la relación existente entre este trastorno crónico y formas más pasajeras. Parece haber un subconjunto de pacientes con una prognosis favorable cuya hipocondria es de corta duración.

Se desconoce la prevalencia de las preocupaciones hipocondriacas en la población general. Dado que la mayoría de las medidas están basadas en escalas continuas, la distinción entre casos de hipocondría y no-casos es una cuestión de grado. Kellner (1985) estimó que la hipocondria oscila del 3 al $13 \%$ en diferentes comunidades y que las preocupaciones acerca de la enfermedad, que van de preocupaciones racionales a miedos constantes e incapacitantes, se presentan en el $10-20 \%$ de la gente normal. Barsky et al. (1990a) informaron de una prevalencia a los seis meses de entre el 4.2 y el $6.3 \%$ en una muestra de pacientes externos de medicina general, según los criterios para el diagnóstico de la hipocondria del DSM-III (American Psychiatric Association, 1987).

Es evidente que la ansiedad por la salud puede oscilar entre ser un fenómeno pasajero o perdurable, y Tyrer et al. (1990) han postulado que algunos pacientes hipocondríacos presentan un trastorno de personalidad más que un trastorno del estado mental. Utilizando un instrumento de investigación estandarizado encontraron que el $8.6 \%$ de un grupo de pacientes con trastornos afectivos presentaban este tipo de trastorno de personalidad. Resulta obvio que se requieren más datos provenientes de estudios longitudinales para establecer los factores de riesgo de estos tipos diferentes de hipocondría.

Los sintomas hipocondriacos son habituales en la depresión (como hipocondría secundaria) $y$, del mismo modo, los sintomas depresivos y la desmoralización intensa son habituales en la hipocondria. Existe una creciente evidencia acerca de elevadas tasas de comorbilidad tanto con problemas de pánico como con problemas obsesivos (Noyes et al., 1986; Salkovskis, Warwick y Clark, 1989; Salkovskis y Clark, 1993). Barsky et al. (1990b) compararon pacientes con "hipocondria pasajera" con pacientes hipocondriacos según criterios del DSM-III (síntomas presentes durante al menos 6 meses). Encontraron que el grupo "pasajero" presentaba menos trastornos psiquiátricos y más morbilidad médica que aquellos con hipocondria $y$, comparados con una muestra de controles no hipocondríacos, el grupo con "hipocondrla pasajera" presentaba más trastornos psiquiátricos, más trastomos de personalidad, más trastomos médicos $\theta$ informaban de ser más conscientes acerca de sus sensaciones corporales.

En el pasado se han utilizado multitud de estrategias psicoterapeúticas para el tratamiento de la hipocondrla, a pesar de ser considerada por muchos como una condición crónica intratable. Cuando se aplicaba un tratamiento, éste estaba basado en las teorías dominantes acerca de la hipocondria, que inclulan motivaciones subconscientes, ganancias primarias o secundarias, y la necesidad de permanecer enfermo (Kellner, 1986). Estudios no controlados más recientes de los métodos modernos de tratamiento han mostrado, sin embargo, que el resultado es mucho mejor de lo que se pensaba (Kellner, 1983; Pilowsky, 1968; Salkovskis y Warwick, 1986; Warwick y Marks, 1988). 
En los últimos 15 años uno de los temas recurrentes en el tratamiento de la hipocondria ha sido el papel que desempeña el hecho de proporcionar tranquilización por una parte, y reducir la evitación ( $y$, por lo tanto utilizar estrategias de exposición y de cambio de creencias) por otra. Estas dos estrategias aparentemente incompatibles han sido recomendadas para el tratamiento de la hipocondria. La cuestión de ofrecer tranquilización ha sido discutida por muchos autores en el pasado incluyendo Gillespie (1928) y Kelliner (1982), mientras que en el caso de la exposición (particularmente cuando se acompaña de una demostración especifica al paciente de que el alivio de la ansiedad producido por la tranquilización es pasajero) es un desarrollo más reciente derivado del tratamiento del trastorno obsesivo-compulsivo (véase Salkovskis y Warwick, 1986; Warwick y Marks, 1988).

En un trabajo que trataba esta cuestión, Kellner (1992) reconoció que la exposición era, con toda probabilidad, el tratamiento más eficaz para los pacientes con una fobia especifica a la enfermedad pura o predominante (personas que fundamentalmente tienen miedo de llegar a estar enfermas). En aquellos pacientes con convicción de enfermedad (personas que creen que están enfermas) Kellner apostaba por un proceso de "persuasión" que implicaba reatribución de los síntomas, explicación, educación, y técnicas cognitivas. Kellner llegó a decir, sin embargo, que las "estrategias de tratamiento incluyen el examen fisico repetido cuando el paciente teme que ha contraido una nueva enfermedad (...) y tranquilización continua.". Parece probable que ésta sea una estrategia inútil para los pacientes que cumplen los criterios del DSM-III dado que, por definición, para estos pacientes "el temor o la creencia persiste a pesar de la tranquilización médica y provoca deterioros en el funcionamiento social u ocupacional".

Warwick (1992) ha sugerido la posibilidad de que Kellner utilizara tranquilización consistente principalmente en proporcionar nueva información al paciente, más detallada y mejor presentada que la tranquilización médica estándar. Este debate subraya la necesidad urgente de una cuidadosa definición del término tranquilización. Como se indicó anteriormente, la tran- quilización puede ofrecerse bajo formas diversas, algunas de las cuales serian útiles, y otras podrian, de hecho, incrementar la ansiedad. Es posible incluso que la ansiedad por la salud pueda estar siendo mantenida debido a la tranquilización.

Como parte de un estudio que informaba del éxito en el tratamiento de dos casos, empleando elementos de exposición y de prevención de la respuesta, Salkovskis y Warwick (1986) demostraron que existe una similitud funcional entre la búsqueda de tranquilización y la ritualización obsesiva (cf. Rachman, de Silva y Roper, 1976). En otra serie de casos de pacientes con temores referentes al SIDA (Miller et al., 1986), siete casos mostraron una mejoria significativa tras el tratamiento cognitivo-comportamental. Este estudio y trabajos más recientes en los enfoques cognitivo-comportamentales para los trastornos de pánico y obsesivo-compulsivo, hacen pensar que los tratamientos cognitivo-comportamentales pueden ser útiles en la hipocondria, dada su adecuada fundamentación térica. Algunas de las diferencias clave entre las rumiaciones obsesivas y las preocupaciones mórbidas ya han sido identificadas por Rachman (1974). Muchas de estas consideraciones pueden aplicarse a la hipocondría, la cual adopta a menudo una forma similar a la del pensamiento obsesivo, pero sin la percepción de ser carente de sentido.

\section{TEORÍAS DE LA ANSIEDAD POR LA SALUD Y LA HIPOCONDRIAA}

No hay evidencia empírica que apoye las numerosas conceptualizaciones psicodinámicas de la hipocondría. Estas tienen un interés únicamente histórico (Kellner, 1985). Estrechamente relacionada, y actualmente más influyente, que los conceptos psicoanalíticos es la noción de "somatización" como proceso. Se ha sugerido que algunas personas son incapaces de expresar el malestar de forma personal y socialmente aceptable, y que el malestar, por lo tanto, emerge en forma de sintomas somáticos.

El mecanismo generalmente implicado en la somatización como proceso es similar al del modelo hidraúlico central del psicoanálisis. La idea de un proceso implícito de enfermedad psicológica subyacente no está respaldada por los resultados de la investigación. Sin embargo, 
los pacientes con tendencia a somatizar informan de malestar psicológico (Simon y Von Korff, 1991). Más importante aún es que esta perspectiva "hidraúlica" aparta la atención clínica e investigadora de la reacción de la persona a los síntomas que de hecho está experimentando, dirigiéndola a algún presunto, pero no detectado, nivel "más profundo" de malestar. Ésto contrasta vivamente con la perspectiva cognitiva en la que la interpretación y malinterpretación de los sintomas son consideradas centrales en la experiencia de malestar y ansiedad, y donde los procesos como la atención selectiva, el arousal psicofisiológico y los cambios en la conducta impulsados por las creencias son considerados cruciales en el mantenimiento del malestar (véase a continuación).

Se asume que la interpretación errónea de las sensaciones corporales resulta importante no sólo debido al desarrollo de la teoria cognitiva, sino también porque forma parte de la definición de hipocondria. Actualmente, hay datos experimentales que demuestran que los pacientes hipocondriacos difieren de los pacientes ansiosos y de las personas normales, tanto en su percepción como en su tendencia a malinterpretar las sensaciones corporales normales (Salkovskis y Clark, 1993; Salkovskis, 1990). Otro conjunto de factores cognitivos se refiere a las conductas de comprobación y de búsqueda de tranqui-lización tan prominentes en muchos pacientes con hipocondria grave.

\section{Un enfoque cognitivo-comportamental}

La hipótesis cognitiva de la ansiedad por la salud y la hipocondria está basada en la noción central de que los signos y síntomas corporales son percibidos como más peligrosos de lo que son en realidad, y en la creencia de que una enfermedad concreta tiene mayor probabilidad de desarrollarse y/o de ser más grave de lo que realmente es (Salkovskis, 1989; Salkovskis y Warwick, 1986; Warwick y Salkovskis, 1989). Al mismo tiempo, es probable que el paciente se perciba a sí mismo como incapaz de impedir la enfermedad y de afectar su curso, es decir, se considera totalmente falto de medios eficaces para afrontar la amenaza percibida. El análisis cognitivo general de la relación entre la amenaza percibida y la experiencia de ansiedad se aplica aqui sin dificultad, es decir,

\begin{tabular}{|c|c|c|c|}
\hline & $\begin{array}{l}\text { Probabilidad } \\
\text { percibida } \\
\text { de enfermedad }\end{array}$ & $\mathbf{x}$ & $\begin{array}{l}\text { Coste percibido, } \\
\text { horror y peso de } \\
\text { la enfermedad }\end{array}$ \\
\hline & $\begin{array}{l}\text { Capacidad } \\
\text { percibida de } \\
\text { poder afrontar } \\
\text { la enfermedad }\end{array}$ & + & $\begin{array}{l}\text { Percepción del grado } \\
\text { en el que ayudarán } \\
\text { los factores externos } \\
\text { (factores de rescate) }\end{array}$ \\
\hline
\end{tabular}

Una implicación crucial de esta descomposición de factores cognitivos es que es posible estar muy ansioso acerca de la salud con una probabilidad relativamente baja de enfermedad real, dada la relativamente alta percepción del horror que supone estar enfermo (por ejemplo, personas que creen que tener cáncer tendrá como resultado quedarse paralizados por el dolor o incapacitados, volverse fisicamente repulsivos, ser rechazados y abandonados por aquellos que aman y estar, en general, deshumanizados). Además, un patrón de este tipo emparejado con una alta probabilidad percibida de tener una enfermedad es probable que resulte en niveles muy extremos de ansiedad. Los cuatro factores han de ser tenidos en cuenta, tanto en la formulación como en cualquier intervención de tratamiento.

En términos del desarrollo de la ansiedad por la salud como un problema grave, se propone que el conocimiento y las experiencias pasadas de enfermedad (en uno mismo o en los demás) lleva a la formación de supuestos específicos acerca de los sintomas, la enfermedad y las conductas de salud. Estos supuestos se aprenden a partir de diversas fuentes, particularmente, experiencias tempranas y sucesos acontecidos en el círculo social del paciente, y a partir también de los medios de comunicación. La experiencia previa de salud-enfermedad de los pacientes y sus familias y la experiencia previa de una atención médica insatisfactoria pueden ser importantes (véase Bianchi, 1971). Un factor adicional es la información reseñada en los medios de comunicación. Un ejemplo notable es el proporcionado por la importante afluencia de casos de "fobia al SIDA" (Miller et al., 1985; Miller at al., 1988) de los que se tuvo conocimiento tras la reciente campaña de publicidad masiva acerca de este tópico.

Ejemplos de supuestos potencialmente problemáticos son: "los cambios corporales son normalmente un signo de enfermedad grave 
porque cada síntoma ha de tener una causa física identificable", o "si no vas al médico tan pronto como notes cualquier cosa inusual luego será demasiado tarde". Otras creencias se relacionan con debilidades personales especificas y enfermedades concretas, por ejemplo: "en mi familia hay problemas de corazón", o "tengo los pulmones débiles desde que era un bebén. Tales creencias pueden ser una fuente constante de ansiedad y/o pueden ser activadas en individuos vulnerables por incidentes criticos.

Estos supuestos pueden llevar también al paciente a atender selectivamente la información que parece confirmar la idea de que tiene una enfermedad, e ignorar selectivamente o descartar la evidencia indicativa de buena salud. Asi pues, estos supuestos concretos conducen a menudo a un sesgo confirmatorio en el pensamiento del paciente, una vez que el incidente crítico ha resultado en la malinterpretación de los sintomas y signos corporales como indicadores de enfermedad grave. Las situaciones que constituyen incidentes criticos $y$ activan supuestos previamente latentes incluyen: sensaciones corporales no familiares, oir detalles de la enfermedad de un amigo de edad similar, o recibir información nueva acerca de la enfermedad. Además, a consecuencia del aumento de la vigilancia que surge de la ansiedad se repara en otrás sensaciones corporales. En pacientes que llegan a estar especialmente ansiosos acerca de su salud, tales situaciones están asociadas a pensamientos que representan interpretaciones personales catastróficas de las sensaciones o signos corporales. Las interpretaciones catastroficas pueden, a su vez, conducir a uno de estos dos patrones de ansiedad. Si las sensaciones o signos no aumentan como resultado de la ansiedad (como consecuencia del arousal autonómico), o bien el paciente no contempla la catástrofe temida como inmediata, entonces la reacción será de ansiedad hipocondriaca acerca de la salud con los correlatos cognitivos, comportamentales, fisiológicos y afectivos detallados en la figura 1 (ejem. "Los dolores de mi estómago quieren decir que tengo un cáncer no detectado"). Por otro lado, si los sintomas que son malinterpretados son aquellos que ocurren como parte del arousal autonómico inducido por la ansiedad, y la interpretación es que los síntomas son los signos de una catástrofe inmediata (ejem. "Estas palpita- ciones quieren decir que estoy teniendo un ataque al corazón en este momento"), el resultado será el consiguiente incremento inmediato en los sintomas. Si este proceso continúa, la respuesta más probable será un ataque de pánico (Clark, 1988; Salkovskis, 1988). A pesar de las diferencias en el tipo de sintomas y en el curso temporal de la enfermedad temida, la ideación en el pánico y la hipocondria es similar y ambas presentaciones a menudo se superponen (véase Noyes et al., 1986).

\section{Factores que mantlenen la ansiedad por la salud}

Una vez que la ansiedad acerca de la salud se ha desarrollado, otros mecanismos pueden estar implicados en el mantenimiento del problema.

1. La ansiedad acerca de asuntos de salud y los síntomas en sI mismos es probable que resulte en arousal fisiológico. Los pacientes pueden entonces malinterpretar los sintomas autonómicos incrementados como una prueba adicional de enfermedad fisica.

2. La atención selectiva a la información relacionada con la enfermedad, tales como la percepción de cambios corporales normales (ej. distensión gástrica después de comer) o rasgos corporales no detectados previamente (ej. piel con manchas) es, a menudo, importante. Este aspecto de focalizar la atención instigado por las preocupaciones acerca de la salud, hace que la persona se dé cuenta de ligeras variaciones corporales en momentos en que las ideas acerca de la enfermedad ya están presentes. dando lugar a un sesgo en el sentido de reparar en información que resulta consistente con las preocupaciones acerca de la enfermedad y con los sesgos confirmatorios preexistentes.

3. La conducta encaminada a evitar, comprobar o excluir totalmente las enfermedades fisicas (ej. evitar el esfuerzo físico excesivo o el contacto con la enfermedad, leer textos médicos, realizar consultas médicas con frecuencia, comprobaciones corporales, manipulaciones e inspecciones) mantendrán la ansiedad mediante el incremento de los síntomas y la preocupación (esta última actua de modo similar al que se puede apreciar en los problemas obsesivos). No obstante, al contrario de lo que ocurre con las interpretaciones erróneas más inmediatas 
de los pacientes con pánico, las falsas interpretaciones hipocondríacas permiten al paciente más posibilidades de buscar seguridad mediante sus intentos de obtener una definición médica de la amenaza percibida a su salud.

La conducta que se produce como consecuencia de la ansiedad puede incrementar la misma a través de varias formas. Por ejemplo, puede mantener la atención centrada en los temores acerca de la salud $y$, por lo tanto, dar lugar a la elaboración de estos temores, aumentar el abanico y el alcance de las interpretaciones catastroficas, e impedir la desconfirmación de las catástrofes temidas (Salkovskis, 1991, 1996; Salkovskis, Clark y Gelder, 1995).

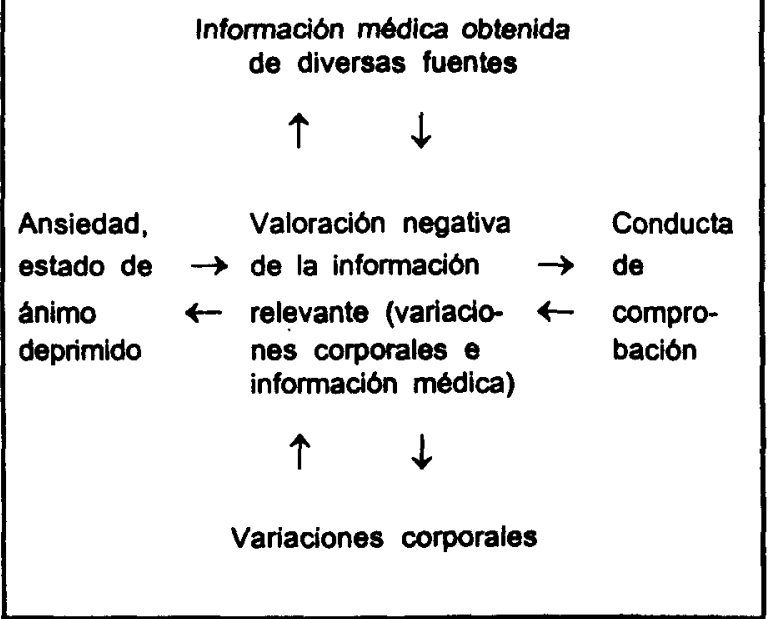

Figura 1: Factores hipotéticos de mantenimiento en la ansiedad por la salud

Estos mecanismos pueden sustentar la preocupación por la salud y exacerbar los sintomas hipocondriacos como se muestra en la figura 1. Consistentes con un enfoque dimensional basado en la vulnerabilidad cognitiva aumentada por mecanismos cognitivos $y$ comportamentales, son los ejemplos de estos procesos que pueden verse en cualquier individuo que tenga un mayor acceso a información nueva referente a los riesgos para la salud (ej. los estudiantes de medicina que perciben síntomas de las enfermedades que están estudiando, reacciones del público a la información ofrecida por los medios de comunicación acerca de nuevos peligros médicos, pacientes que sufren - esperan los resultados de pruebas diagnósticas). La atención selectiva y la conducta de comprobación sirven para mantener la ansiedad a largo plazo, del mismo modo que lo hace la neutralización en el trastorno obsesivo (Salkovskis y Warwick, 1986).

\section{TRATAMIENTO COGNITIVO- COMPORTAMENTAL}

De este modelo cognitivo-comportamental se sigue que el tratamiento de la hipocondrla ha de implicar el llegar a una comprensión compartida con el paciente acerca de la base psicológica de su problema. Ésto es crucial porque, al principio de la terapia, estos pacientes creen que es probable que estén sufriendo una enfermedad grave o que ésta representa una amenaza para su vida. Si esta creencia se mantiene firmemente, es improbable que el paciente se comprometa en un tratamiento con base psicológica (o psiquiátrica). No es sorprendente que el paciente hipocondríaco que cree que tiene una enfermedad coronaria o cáncer sea reacio a tratarlos por medios psicológicos. Más bien, el paciente busca solucionar su problema mediante estrategias tales como obtener la ayuda médica apropiada. Es, por lo tanto, necesario que en las primeras fases del tratamiento se ayude a los pacientes a ver que puede haber una explicación alternativa para los problemas que están experimentando. Se les presenta un modelo cognitivo, basado en sus propias idiosincrasias, que ofrece una visión bastante diferente y menos amenazante de sus problemas. Es decir, no es que ellos tengan cáncer sino que están preocupados por y creen que pueden tener cáncer. Para que el tratamiento sea eficaz se desprende que es crucial que los pacientes estén de acuerdo en que las estrategias terapéuticas deben estar dirigidas a la reducción de tales preocupaciones en lugar de los infructuosos intentos de reducir el riesgo.

En esta fase del tratamiento (compromiso) las dos explicaciones posibles para los problemas del paciente son consideradas conjuntamente, en vez de considerarlas como alternativas mútuamente excluyentes. Se invita al paciente a considerar cómo las dos posturas alternativas se ajustan a su experiencia. Una vez que el terapeuta y el paciente están de acuerdo 
en las dos alternativas, la terapia continúa como una evaluación de los méritos relativos de las dos posturas. Se discute y se revisa en detalle la evidencia a favor y en contra

A menudo la discusión alcanza un punto en el que debe buscarse información adicional que no está actualmente disponible para el paciente. Es aquí donde entran en escena los experimentos comportamentales. Los experimentos comportamentales son ejercicios de recogida de información que ayudan al paciente a alcanzar conclusiones acerca de las creencias que mantiene. Por ejemplo, un paciente puede haber notado que su dolor en el pecho tiende a empeorar cuando hace deporte. Durante la discusión, el paciente no está seguro si ésto sucede antes, durante o después del ejercicio. Con el proposito de someterlo a prueba, terapeuta y paciente dan un enérgico paseo por el hospital haciendo evaluaciones repetidas de la intensidad del dolor en el pecho. Existe una constante interacción entre la formulación cognitivo-comportamental esbozada por paciente y terapeuta, la discusión acerca de cómo la experiencia del paciente se ajusta a esa formulación, y la generación de experiencias nuevas e informativas empleando los experimentos comportamentales para iluminar más el modelo.

Los elementos cognitivos y comportamentales están entrelazados, pero el principio orientador es siempre permitir a los pacientes considerar y adoptar creencias más útiles y menos atemorizantes que aquellas que hablan tenido que soportar previamente.

En el tratamiento de la ansiedad por la salud la idea es que los factores que previamente desencadenaban la ansiedad y la incomodidad pueden seguir estando presentes. No obstante, la terapia tiene el efecto de modificar el significado de las variaciones físicas al nivel experimentado por la mayoria de otras personas que no padecen un trastorno de ansiedad de este tipo. No se lleva a cabo ningún intento para disminuir el número de sensaciones o variaciones corporales experimentadas, y cualquier intento por parte del paciente para efectuar tal reducción se reta sobre las bases de esas creencias que lo motivaban. No obstante, un afortunado y deseable efecto secundario de la terapia cognitiva es que, normalmente, se produce una disminución real en tales síntomas.
Nótese que la persona "normal" no busca constantemente controlar los síntomas, el control tiende a ser indirecto porque no hay consecuencias negativas graves derivadas del fracaso en el control de los sintomas.

La meta del tratamiento es ayudar al paciente a identificar cómo opera su problema, más que descartar la enfermedad física. Este proceso requiere que el terapeuta reconozca desde el príncipio que los sintomas experimentados por el paciente realmente existen y que el tratamiento se dirige a proporcionar una explicación satisfactoria de estos sintomas. Para alcanzar esta meta, las sesiones de tratamiento no deberian nunca llegar a ser combativas: el realizar preguntas y la colaboración conjunta entre paciente y terapeuta son las técnicas clave. El logro de esta meta se ve enormemente simplificado si el terapeuta recuerda que las creencias del paciente están basadas invariablemente en la evidencia que él encuentra convincente. El buen terapeuta cognitivo-comportamental comienza el proceso de desafiar las creencias descubriendo las observaciones que el paciente cree que son prueba de enfermedad y trabajando después, en colaboración con el paciente, sobre esta base. Por último, dado que, por definición, los pacientes hipocondriacos tienden a malinterpretar la información relevante para su salud, es crucial que el terapeuta compruebe el grado de comprensión del paciente acerca de lo tratado en las sesiones de tratamiento, pidiéndole que haga un resumen y que señale las implicaciones que para él tiene.

\section{El cambio de la conducta y los experimentos comportamentales}

La mayor parte de las conductas implicadas en los problemas somáticos son percibidas por el paciente como teniendo una función preventiva y son, por lo tanto, relativamente dificiles de modificar si no se presta atención a las creencias subyacentes.

\section{Conductas directamente relacionadas con el problema}

Cuando la conducta de enfermedad es notable, las estrategias de tratamiento se dirigen a elicitar y demostrar el papel de las conductas en el mantenimiento de la ansiedad, la preocu- 
pación y la alteración fisiológica. El uso de las preguntas como parte del descubrimiento guiado puede ser útil. La demostración directa es especialmente convincente cuando cambiar la conducta muestra tener un efecto en los síntomas. Paciente y terapeuta diseñan experimentos para (a) someter a prueba la creencia del paciente de que la conducta está "manteniéndole a salvo" de un daño grave y (b) ver si las conductas que el paciente cree que alivian sus sintomas realmente lo hacen. Hacer que el paciente aumente su conducta para demostrar los efectos sobre los síntomas generalmente resulta más útil que los experimentos diseñados para reducir el sintoma (ej. el paciente que está preocupado de que un "nudo en la garganta" significa que tiene cáncer tiende a someterlo a prueba tragando más frecuentemente; el terapeuta y el paciente tragan con frecuencia durante cinco minutos en la sesión y después discuten los resultados).

En muchos casos, las conductas de evitación mantienen la preocupación de los pacientes por la enfermedad impidiéndoles acceder a la información que contradice las interpretaciones negativas de los sintomas.

\section{Tranquilización}

En pacientes con ansiedad por la salud, pueden producirse varias conductas cuyo efecto es el mismo que el de la comprobación obsesiva. Estas conductas de búsqueda de tranquilización centran la atención en las preocupaciones de los pacientes y reducen su ansiedad a corto plazo, pero incrementan la preocupación y otros aspectos del problema a largo plazo (Salkovskis y Warwick, 1986; Warwick y Salkovskis, 1985). Estos pacientes pueden además ganarse la antipatia del personal médico y de los familiares; los psicotera-peutas tampoco son inmunes a las reacciones negativas de la continua búsqueda de tranqui-lización. Ésta incluye peticiones de pruebas diagnósticas fisicas, revisiones físicas, asi como una detallada discusión de los sintomas en un intento de descartar la posible enfermedad. Aunque la mayorla de los pacientes no ansiosos que buscan ayuda médica reaccionan adecuadamente a la tranquilización proporcionada, en la cual la enfermedad es "descartada"; los pacien- tes ansiosos acerca de su salud responden de manera diferente: la tranquilización repetida y "más convincente" rápidamente se convierte en contraproducente, en tanto que el paciente atiende selectivamente y malinterpreta la tranquilización en sí misma. A un paciente, por ejemplo, se le dijo: "Estos dolores de cabeza están causados seguramente por la tensión; si persisten, entonces le enviaré a que se haga una radiografia craneal para tranquilizarle". EI paciente interpretó esto como un signo de que el doctor pensaba que podría tener un tumor cerebral. Los repetidos intentos de "probar" a los pacientes que no están enfermos, sea a través de las pruebas médicas o de la persuasión verbal, es probable que aumenten la ansiedad.

El papel de la búsqueda de tranquilización en el mantenimiento de los problemas de los pacientes se les debe explicar de forma que lo entiendan claramente. De hecho, se invita al paciente a que especifique cuánta y qué tipo de tranquilización sería suficiente para manejar sus miedos durante un período de tiempo largo, por ejemplo, un año. La mayoría de los pacientes dirá que la tranquilización no puede durar tanto, que los efectos, generalmente, se desvanecen en horas o incluso menos, y que el ansia por ser tranquilizados casi siempre recupera el mismo nivel $O$ asume niveles más graves. El terapeuta y el paciente discuten pues el valor de la tranquilización a la luz de ésto. De este modo, el paciente dice al terapeuta que la tranquilización es un modo inútil de tratar con su ansiedad, y ambos pueden continuar discutiendo alternativas a la tranquilización en un trabajo de colaboración conjunta. Esta discusión puede entonces abordarse para considerar formas de cortar la búsqueda de tranquilización y reducir las conductas de comprobación, como parte de la estrategia de disminuir la ansiedad y la preocupación acerca de la salud.

Es útil diseñar un experimento comportamental que demuestre los efectos de la tranquilización alli donde su búsqueda es un rasgo principal de los problemas del paciente (Salkovskis y Warwick, 1986). Este experimento puede funcionar también como una estrategia de compromiso en pacientes reacios a comenzar el tratamiento sin una "prueba definitiva". Se discute y se fija, por ejemplo, una última revisión física antes de que comience el tratamiento psicológico, con la comprensión estricta de que 
es considerada innecesaria para la salud fisica del paciente, pero que puede ser útil para la evaluación psicológica. Se evalúa la ansiedad acerca de la salud, la creencia en pensamientos especificos relacionados con la enfermedad, y la necesidad de tranquilización, de manera regular, en una escala de 0 a 100 en los periodos anterior y posterior a la prueba médica. Si la ansiedad se ha reducido de manera duradera, entonces ésto es útil en cualquier caso. $\mathrm{Si}$, como es mucho más corriente, la ansiedad se reduce sólo brevemente, ésto es utilizado como base para la discusión acerca del modo en el cual la tran-quilización mantiene la ansiedad. La demostración también compromete al paciente en el tratamiento y establece una relación de colaboración. Proporciona una razón lógica para controlar la búsqueda de tranquilización $y$, por lo tanto, ayuda al paciente a tolerar la ansiedad inicial causada por el cambio de conducta. Una estrategia similar es pedir a los pacientes que especifiquen exactamente qué procedimientos les convencerían completamente de que no están padeciendo la enfermedad temida. El terapeuta adopta entonces el papel del escéptico preguntando cosas como: "Si, pero ¿sería esto realmente convincente? ¿Cómo puede estar seguro de que el médico era un verdadero experto en la utilización de la prueba?", etc. Esto sirve para ilustrar que nunca es posible estar seguro de que la enfermedad no está presente, del mismo modo que nunca es posible estar seguro de que un satélite no nos va a caer sobre la cabeza cuando paseamos por la calle. Esta discusión está relacionada con la importancia de la tranquilización en el mantenimiento de la ansiedad, la preocupación y la creencia de enfermedad.

Se debe incluir a la familia y a otras personas implicadas con el paciente en estas discusiones, y se les debe enseñar como manejar las peticiones de tranquilización. Puede utilizarse un juego de roles en el que el paciente pide al familiar que le tranquilice y el familiar contesta en términos previamente acordados ( $y$ sin crítica no-verbal). Por ejemplo, el familiar podria replicar: "Como acordamos en la clínica, no te sirve de ayuda si te tranquilizo al respecto, así que no voy a responderte en absoluto." El familiar entonces o bien se va o habla de cosas que no tengan relación con éso. Excepto como último recurso, para las ocasiones en las que el paciente está especialmente estresado, este tipo de estrategia es de poca utilidad $\sin$ el acuerdo previo del paciente.

Revisión del resultado de la investigación

A pesar del hecho de que la hipocondria haya sido identificada como un sindrome inequivoco hace más de un siglo, no existen datos previos procedentes de ensayos controlados acerca de la eficacia de ningún tipo de tratamiento. A lo largo de las pasadas dos décadas se ha dado cuenta de cierto número de informes de casos, experimentos de caso único y series de casos, indicando que el tratamiento puede ser eficaz. Por ejemplo, Salkovskis y Warwick (1986) informaron del tratamiento de dos pacientes que erróneamente creian que estaban padeciendo enfermedades que representaban una seria amenaza para sus vidas. Para ambos pacientes se estableció una línea base en la cual se recogieron pruebas de los efectos ansiógenos de la tranquilización médica a través del registro en un diario. Se presentó esta información al paciente como el resultado de un experimento comportamental senalando el posible papel de la búsqueda de tranquilización y las conductas de comprobación asociadas. Esta información fue presentada al paciente y la explicación cognitiva de los problemas de los pacientes resulto concordar con las mismas. Los pacientes mostraron una mejoría sustancial y sostenida una vez se comprometieron en el tratamiento. En una serie de casos, que incluian fóbicos a la enfermedad y pacientes con altos niveles de convicción de enfermedad, Warwick y Marks (1988) utilizaron una estrategia basada en la exposición, la prevención de la respuesta $y$ el cambio de creencias que obtuvo un buen resultado. Més recientemente, un tratamiento cognitivo-comporta-mental similar administrado en el contexto de una terapia de grupo resultó ser eficaz en un estudio no controlado con pacientes de hospital general (Stern y Femández, 1991).

Se han llevado a cabo hace poco dos ensayos controlados de terapia cognitiva en la línea de la descrita en este trabajo. En el primero, Warwick, Clark, Cobb y Salkovskis (1996) compararon 16 sesiones de tratamiento cognitivocomportamental con un control lista de espera 
de duración similar. En 23 de las 24 medidas, el grupo tratado mejoró significativamente más que el grupo de lista de espera, con una valoración media de la mejorla realizada por el evaluador del $5 \%$ en el grupo de lista de espera en oposición al $76 \%$ en los tratados con terapia cognitivo-comportamental. Las ganancias del tratamiento se mantuvieron en la mayoria de los casos a los 3 meses de seguimiento. En un segundo estudio, el tratamiento cognitivocomportamental se comparó con un programa terapéutico cuidadosamente diseñado de manejo del estrés y con un control lista de espera. En el momento de escribir este trabajo no se encontraban disponibles todavia todos los resultados de este ensayo, pero los análisis preliminares de los datos realizados para su presentación en una conferencia (con un pequeño número de pacientes sin haber finalizado la terapia y, por lo tanto, no incluídos en el análisis) indican que ambos tratamientos activos son significativamente mejores que la condición de lista de espera. Este resultado no es inesperado en tanto que el manejo comportamental del estrés proporciona a los pacientes una detallada explicación alternativa para sus sintomas (ej. estrés) y un conjunto comprehensivo de técnicas para su manejo basadas en esta explicación alternativa. El análisis definitivo nos permitirá determinar la eficacia relativa de los dos tratamientos a corto y a largo plazo, y el papel de las variables cognitivas en la predicción de la recalda. Un estudio realizado en España ha demostrado también la eficacia de este tipo de tratamiento. En un ensayo que evaluaba la terapia cognitivo-comportamental basada en el enfoque psicoeducativo de Arthur Barsky en personas que padecian ansiedad por la salud (de los que no todos cumplian los criterios diagnósticos para la hipocondria), Avia et al. (1996) encontraron que el tratamiento de grupo era más eficaz que un grupo control lista de espera.

\section{Nuevas direcciones}

Ahora que la eficacia del tratamiento cognitivo-comportamental para la hipocondria grave ha sido establecida en ensayos controla dos, los desarrollos futuros se encuentran en, al menos, dos áreas relacionadas. En primer lugar, han de identificarse los componentes efectivos del tratamiento, especialmente a la luz de la eficacia del tratamiento comportamental para el manejo del estrés. Los desarrollos en esta dirección deberian dar lugar a intervenciones más breves y eficaces. Nuestro grupo está a punto de empezar un estudio para evaluar una versión breve del tratamiento que se ha descrito aqui ( 8 sesiones en lugar de 16). Este estudio será llevado a cabo en el contexto de la atención primaria en vez de en la clínica psiquiátrica, ya que parece que la mayoria de pacientes con ansiedad por la salud son vistos en este contexto y son reacios a acudir a la clínica psiquiátrica. En segundo lugar, se espera que este trabajo que utiliza un modelo cognitivocomportamental pueda ampliarse a la comprensión y tratamiento de la ansiedad como reacción a las pruebas y exploraciones médicas (Salkovskis y Rimes, remitido para publicación). Es probable que este tipo de desarrollo sea cada vez más importante en tanto que las evaluaciones realizadas en toda la población lleguen a ser más ampliamente disponibles y aplicables. Ya hemos encontrado que las medidas del pre-test derivadas de las hipotesis cognitivo-comportamentales eran predictores significativos de reacciones psicológicas negativas a la evaluación de la densidad ósea para la osteoporosis (Rimes y Salkovskis, en preparación). Actualmente estamos planeando aplicar el modelo cognitivo-comportamental a un examen del impacto psicológico de las nuevas técnicas de diagnóstico genético. Se sabe que algunos individuos experimentan importantes reacciones negativas a esta información sobre el riesgo genético (e.g. Lannfelt, Lilius y Basun, 1995), pero ha habido poca investigación acerca de la eficacia de las intervenciones que las personas reciben en estas situaciones. El uso de un modelo cognitivo-comportamental para la comprensión y predicción de la reacción psicológica a la realización de pruebas médicas ofrece la posibilidad de un asesoramiento psicológico específico previo a la prueba, diseñado para prevenir las reacciones negativas y para adoptar tratamientos cognitivos para la ansiedad por la salud y la depresión que permitan aliviar el malestar psicológico posterior a dicha prueba. 


\section{REFERENCIASBIBLIOGRÁFICAS}

American Psychiatric Association (1994). Diagnostic and statistical manual of mental disorders, $4^{\text {th }}$ edition. A.P.A., Washington D.C.

Barsky, A. J. and Klerman, G. L. (1983). Overview: hypochondriasis, bodily complaints and somatic styles. American Journal of Psychiatry 140, 27381.

Barsky, A. J., Wyshak, G., Klerman, G. L. and Latham, K.S. (1990a). The prevalence of hypochondriasis in medical outpatients. Social Psychiatry and Psychiatric Epidemiology, 25, 89-94

Barsky, A. J., Wyshak, G. and Klerman, G. L. (1990b). Transient hypochondriasis. Archives of General Psychiatry 47, 746-752.

Beck, A.T. (1976). Cognitive therapy and the emocional disorders. International Universities Press, New York.

Beck, A.T., Emery, G., and Greenberg, R. (1985) Anxiety disorders and phobias: a cognitive perspective. Basic Books: New York.

Bianchi, G.N. (1971) The Origins of Disease Phobia. Australia and New Zealand Joumal of Psychiatry, 5, 241-257.

Bovd, J.H., Burke, J.D., Gruenberg, E. et al. (1984). Exclusion criteria of DSMIH-psychiatric disorders in primary care, Journal of Psychosomatic Research, 29, 563-569.

Brido,es, K.W., and Goldberg, D.P. (1985) Somatic Presentation of DSM III Psychiatric Disorders in Primary Care. Journal of Phychosomatic Research, 29, 563-569.

Clark, D.M. (1988). A cognitive model of panic attacks. In Panic: psychological perspectivas, (ed. S. Rachman, and J.D. Maser), pp.71-90. Lawrence Erlbaum, Hillsdale NJ.

Cloninger, C. (1986). Diagnosis of somatoform disorders: a critique of DSM III. In Diagnosis and Classification in Psychiatry, (Ed. Tischler, G.), Cambridge University Press, New York.

Gelder, M., Gath, D., and Mayou, R. (1984) Oxford Textbook of Psychiatry, Oxford University Press: Oxford.

Johnston, D.W. (1984). Biofeedback, relaxation and related procedures in the treatment of psychophysiological disorders. In Health care and human behaviour, (ed. A. Steptoe, A. Mathews), pp. 267300. Academic Press, London.

Katon, W. (1984). Panic disorder and somatization. The American Journal of Medicine. 77, 101-6.

Katon, W., Ries, R.K., and Kleinman, A. (1984) The Prevalence of Somatization in Primary Care. Comprehensive Psychiatry, 25, 208-21 1. Kellner 1985; Mayou 1976)

Kellner, R. (1982) Psychotherapeutic strategies in hypochondriasis: a clinical study. American Journal of Psychiatry 36, 146-157.
Kellner, R. (1985) Functional somatic symptoms and hypochondriasis. Archives of General Psychiatry 42,821-833.

Kellner, R. (1985) Functional somatic symptoms and hypochondriasis. Archives of General Psychiatry 42,821-833.

Kellner, R. (1989). Theories and research in hypochondriasis. The $1988 \mathrm{C}$. Charles Burlingame Award Lecture. Hartford, Conn: The Institute of Living.

Kellner, R. (1992). The treatment of hypochondriasis: to reassure or not reassure? International Review of Psychiatry, 4, 71-75.

Kellner, R., Abbott, P., Winslow, W.W., and Pathak, D. (1987) Fears, Beliefs and Attitudes in DSM III Hypochondriasis. Journal of Nervous and Mental Disease, 175 , 20-25.

Kellner, R., Fava, G.A., and Lisansky, J. (1986) Hypochondriacal fears and beliefs in DSM III melancholia. Journal of Affective Disorders, 10, 21-26.

Kenyon, F.E. (1964) Hypochondriasis: a clinical study. British Journal of Psychiatry 110,478-488.

Kenyon, F.E. (1965) Hypochondriasis: A Survey of some Historical, Clinical and Social Aspects. British Journal of Psychiatry, 119, 305-307.

Ladee, G.A. (1966). Hypochondriacal syndromes. North Holland, Amsterdam.

Lang, P.J. (1970) Fear reduction and fear behaviour: problems in treating a construct. In Research in Psychotherapy, Vol IH, (ed. J.M. Schlien), pp.90103. American Psychological Association, Washington D.C.

Lannfelt, L., Axelman, K., Lilius, L. \& Basun, H. (1995). Genetic counselling in a Swedish Alzheimer family with amyloid precursor protein mutation. (Letter). American Journal of Human Genetics, 56, 332-335.

Lipowski, Z. J. (1986a). Psychosomatic concepts in historical perspective. In Proceedings of the $15^{\text {th }}$ European conference on psychosomatic research, (ed. J. H. Lacey, and D. A. Sturgeon), pp. 1-5. John Libbey, London.

Lipowski, Z.J. (1986b). Somatization: a borderland between medicine and psychiatry. Canadian Medical Association Journal 135, 609-14.

Lipowski, Z.J., (1988). In in-patient programme for persistent somatizers. Canadian Journal of Psychiatry, 33, 275-278.

Mayou, R. (1976) The Nature of Bodily Symptoms. British Journal of Psychiatry, 129,55-60.

Miller, D., Green, J., Farmer, R., and Carroll, G. (1985) A "pseudo-AIDS" syndrome following from fears of AIDS. British Journal of Psychiatry, 146, 550551.

Miller, D., Acton, T.M.G., and Hedge, B. (1988) The worried well: their identification and management. 
Journal of the Royal College of Physicians, 22 , 158-165.

Noyes, R., Reich, J., Clancy, J. And O'Gonnan, J.W. (1986). Reduction in hypochondriasis with treatment of panic disorder. British Journal of Psychiatry, 149, 631-635.

Pilowsky, I. (1967) Dimensions of Hypochondriasis. British Joumal of Psychiatry, 113, 89-93.

Pilowsky, 1. (1 968). The response to treatment in hypochondriacal disorders. Australian and New Zealand Journal of Psychiatry, 2, 88-94.

Pilowsky, I. (1970) Primary and Secondary Hypochondriasis. 273-285.

Pilowsky, I. (1984). Hypochondriasis. In Handbook of psychiatry (Vol.4). Cambridge University Press, Cambridge.

Rachman, S.J. (1974) Some similarities and differences between obsessional ruminations and morbid preoccupations. Can. Psychiat. Ass. J., 18, 71-73.

Rachman, S.J., Hodgson, R. (1980). Obsessions and compulsions. Prentice Hall, Englewood Cliffs NJ.

Rachman, S.J., de Silva, P. and Roper, G. (1976) The spontaneous decay of compulsive urges. Behaviour Research and Therapy, 14, 445-453.

Rimes, K.A. \& Salkovskis, P.M. (In preparation). Reactions to bone density screening in -women with high and low levels of pre-existing health anxiety.

Rov, A. (1979). Hysteria: a case note study. Canadian Journal of Psychiatry, 24, 157-160.

Ryle, J.A. (1947) Nosophobia. Journal of Mental Science, 94, 1-17.

Salkovskis, P.M. (1988). Phenomonology, assessment and the cognitive model of panic attacks. In Panic: Psychological views, (Eds. Rachman, S.J., Maser, J.), Lawrence Erlbaum.

Salkovskis, P.M. (1989). Obsessions and compulsions. In Cognitive therapy: a clinical casebook, (ed. J. Scott, J.M.G. Williams, and A.T. Beck) Routledge, London.

Salkovskis, P.M. (1989). Somatic problems. In Cognitive-behavioural approaches to aduit psychological disorder: a practical guide, (Eds. Hawton, K., Salkovskis, P.M., Kirk, J.W., Clark, D. M.), Oxford University Press, Oxford.

Salkovskis, P.M. (1990). The nature of and interaction between cognitive and physiological factors in panic attacks and their treatmentw. Unpublished Ph D thesis, University of Reading.

Salkovskis, P.M. (1991). Tile importance of behaviour in the maintenance of anxiety and panic: a cognitive account. Behavioural Psychotherapy, 19, 6-19.

Salkovskis, P.M. (1994). Principles and practice of cognitive-behavioural treatment of obsessional problems. Praxis der Klínischen Verhaltensmedizin und Rehabilitatioli, 7, 113-120.

Salkovskis, P.M., Clark, D.M. (1993) Panic disorder and hypochondriasis. Advances in Behaviour Research and Therapy, 15, 23-48.

Salkovskis, P.M. \& Rimes, K.A. (Submitted). Psychological factors, medical screening and the advance of human genetics.

Salkovskis, P.M., Warwick, H.M.C. (1986). Morbid preoccupations, health anxiety and reassurance: a cognitive-behavioural approach to hypochondriasis. Behaviour Research and Therapy. 24, 597-602.

Salkovskis, P.M., Warwick, H.M.C., Clark, D.M. (1990). Hypochondriasis. Paper for DSM IV working' groups.

Salkovskis, P.M. (1995) Treatment of Hypochondriasis. Paper presented at the World Congress of Behavioural and Cognitive Therapies, Copenhagen, July 1995. .

Simon, G.E. and von Korff, M. (1991). Somatization and psychiatric disorder in the NIMH EpIdemiologic Catchment Area Study. American Journal of Psychiatry, 148, 1494-1500.

Tyrer, P., Fowler-Dixon, R. and Ferguson, B. (1990). The justification for the diagnosis of hypochondriacal personality disorder. Journal of Psychosomatic Research, 34, 637-642.

Warwick, H.M.C. (1992). Provision of appropriate and effective reassurance. International Review of Psychiatry, 4, 76-80.

Warwick, H.M.C. (1993). A controlled trial of cognitive therapy for hypochondriasis. Paper presented at EABCT conferencey London, Septembe, 1993.

Warwick, H.M.C., Clark, D.M., Cobb, A., and Salkovskis, P.M. (1996): A controlled trial of cognitive-behavioural treatment of hypochondriasis. British Joumal of Psychiatry.

Warwick, H.M.C. y Marks, I.M. (1988). Behavioural treatment of illness phobia. British Journal of Psychiatry, 152, 239-241.

Warwick, H.M.C. y Salkoyskis, P.M. (1985). Reassurance. British Medical Joumal, 290. 1028.

Warwick, H.M.C. y Salkoyskis, P.M. (1989). Hypochondriasis. In Cognitive therapy in clinical practice, (ed. Scott, J., Williams, J.M.G., Beck, A.T.), Gower, London.

Weisenberg, M. (1987). Psychological intervention for the control of pain. Behaviour Research and Therapy, 25, 301-312.

World Health Organisation (1978). Mental disorders: Glossary and guide to their classification in accordance with the tenth revision of the international classification of diseases. W.O.H. Geneva. 